\title{
A PERFORMANCE IMPROVEMENT SCHEME OF CONTENT CENTRIC NETWORKING OVER MULTI-SOURCE CONTENT DISTRIBUTION
}

\author{
ONG MAU DUNG, BUI THU CAO \\ Industrial University of Ho Chi Minh City; \\ ongmaudung@iuh.edu.vn,buithucao@iuh.edu.vn
}

\begin{abstract}
Nowadays, Content Centric Networking (CCN) becomes one of the important technologies for enabling future network. In $\mathrm{CCN}$, the contents are cached along the reverse path from the producer to the consumer, and they may be reused for many times without fetched from the original producer. In such kind of operation, the $\mathrm{CCN}$ improves network performance by reducing redundant transmissions of popular contents. However, the volume of the content store (CS) located in the gateway/router is constrained, and much smaller than the size of existing Internet contents which would go through the gateway/router, when rich media applications are considered. In order to solve this issue, this paper proposes a novel Multi-Source Content Centric Networking (MS-CCN) scheme by leveraging the concept of Multi-Source Mobile Streaming $\left(\mathrm{MS}^{2}\right)$. In the MS-CCN model, the caching of each content is not limited in the single server anymore as in conventional CCN model, instead, each content is fragmented and distributed to multiple servers over a large scale network. After experiencing disjoint multi-paths, various content fragments are concatenated at client side. The OPNET Modeler simulation results show that the MS-CCN scheme significantly outperforms the original CCN scheme in terms of network utilization and user's Quality of Experiment (QoE).
\end{abstract}

Keywords. Content centric networking, caching, multi-streaming, network traffic offload.

\section{INTRODUCTION}

Recently, the global data traffic forecast from Cisco has unveiled two important trends relative with mobile networking [1]. Firstly, the amount of global mobile data traffic increases exponentially from year 2014 to 2019 . Secondly, mobile video traffic dominates Internet traffic by occupying $50 \%$ percentages of whole traffic in 2012, then 55\% percentages by the end of 2014, indicating that mobile video traffic impacts on Internet traffic today. Usually, single-source single-path (SSSP) routing is used between end user and server due to its simplicity. However, facing the sheer volume of rich video applications, the traditional SSSP routing will cause the bandwidth of Internet backbone insufficient to keep up with the Quality of Service (QoS) requirement for the huge number of mobile users.

Content Delivery Network (CDN) are capable to delivering better end-to-end performance than original clients-server by deploying in widely a large number of Autonomous System (AS). CDN includes some center servers and the number of edge servers placed in wide geographic locations. Using $\mathrm{CDN}$, the request content come from users will be redirected to the nearest edge server to solve the backbone network bottleneck and provide better quality of service. However, with the exponential growth of Internet traffic, especially video traffic, the $\mathrm{CDN}$ requires very high cost for large storage in the edge servers. Besides CDN, cloud computing provides elastic infrastructure and pay-as-you-go. These characteristics make cloud computing become a suitable solution for the drawback of CDN about large storage [2].

In comparison, multi-source single-path (MSSP) proposed for the better utilization of the overall possible Internet links. By splitting of data packets among different routing paths, the average packet drop rate on each path is smaller in case of network congestion. In [3], a multi-source mobile streaming ( $\left.\mathrm{MS}^{2}\right)$ architecture is proposed to further alleviate the impact of network congestion on mobile streaming services, by efficiently utilizing the available network resources through an effective rate allocation scheme among multiple sources that collaborate to stream the same content in a complementary manner. However, the total bits transferred between server side and end user side is not reduced for all above situations, e.g. SSSP, MSSP, and $\mathrm{MS}^{2}$. 
Related with the exponential growth of traffic, a skewness of popularity content characteristic was discussed in [4], that is to say, a few most popularity contents are often queried by the huge number of end users. Therefore, it is critical to design an effective way to minimize the duplicate content deliveries to save bandwidth and offloading server traffic. In order to alleviate the bandwidth problem and concerned the skewness of popularity content, content centric networking $(\mathrm{CCN})$ is proposed to effectively distribute popular data content to a huge number of users [5]. To maximize the probability of sharing with minimal upstream bandwidth demand and lowest downstream latency, routers/gateways should keep all arrived contents as long as possible. Furthermore, reducing traffic load by in-network caching can enhance the mobile network with higher energy efficiency and toward the evolution to the "green" mobile network.

In this paper, following the $\mathrm{MS}^{2}$ architecture and the background of the $\mathrm{CCN}$, we integrate the $\mathrm{MS}^{2}$ mechanism to CDN and CCN architecture, dulled by Multi-Source CDN (MS-CDN) and Multi-Source CCN (MS-CCN), respectively. Our OPNET Modeler simulation [6] results prove that the CCN is a good revolution for existing challenges of traditional IP network. And with the same network configuration, the MS-CCN outperforms than the original CCN and MS-CDN with lower round trip time, lower effect from the Internet bottleneck links and offloading servers.

The remainder of this paper is structured as follows. Section 2 highlights some recently research work pertaining to multi-path, multi-source streaming techniques, and overview the $\mathrm{MS}^{2}$ model operation. Section 3 describes the MS-CDN, MS-CCN network architectures and evaluates the precision of the simulation. Section 4 further presents the MS-CCN improvement based on the network model from Section 3. Finally, the paper concludes in Section 5.

\section{RELATED WORK}

\subsection{CCN overview}

Streaming services from nearby nodes or routers goes back to a decade or longer [7]. Content Centric Networking came to play to organize such an efficient streaming based on smart caching of popular content nearby the requesting users. The CCN model presents a simple but effective communication. In $\mathrm{CCN}$, two types of packets are envisioned to identify a content, which is typical hierarchical and human readable. They are namely interest packet (IntPk) and data packet (DataPk). CCN nodes maintain three data structures: Forwarding Information Base (FIB), Pending Interest Table (PIT) and Content Store (CS). Once a CCN node receives an IntPk, it looks up its CS. If an appropriate content is found as known as hitting content, some DataPks will be sent for a request, otherwise the IntPk will be checked in PIT. PIT keeps track of unsatisfied IntPks. After PIT creates a new entry for an unsatisfied IntPk, the IntPk is forwarded to upstream towards a potential content source based on FIB's information.

A returned DataPk will be sent to downstream and stored on CS. In general, a content is cached at routers for a certain time. When the "caching" deadline expires, the content is removed to cope with the limited size of content storage. When CS is about to get full or receive a new content, it stores the new content according to the underlying replacement policy to leave space for the new content. Least Recently Used (LRU), Least Frequently Used (LFU) and First In First Out (FIFO) are few notable examples of replacement policies for $\mathrm{CCN}$.

\subsection{Multi-streaming}

For the actual delivery of streaming services, a wide library of research works has been conducted. Many research works have considered the delivery of multimedia on multiple routing paths $[8,9,10]$.

The concept of multi-path routing has been also investigated in the context of CCN. In [11], some disadvantages attributed to the original $\mathrm{CCN}$ routing scheme are highlighted. That is, the best-face routing (BFR) mechanism is used to find the highest suitable routing path for the IntPk uplink routing and the DataPk download. Because others possible faces are unused, the BFR mechanism is become unbalance load and the load of the best face with its repository is easy overloaded. To overcome with this limitation, the multi-path routing scheme is proposed, whereby different repositories are enable to retrieve from different chunks of one content simultaneously. 
Similarly, in [12], the potential of multi-path communications in CCN is explored. In order to compare performance between multi-path and single-path schemes, some important metrics are used such as: round trip time (RTT), throughput and download time. The original Round Robin distribution mechanism is used to split the traffic strategy. Moreover, the skewness popularity of content, e.g. Zipf based popularity model, is discussed with adopting replacement policies such as LRU, FIFO, and random replacement.

\subsection{A basic MS2 model}

The example $\mathrm{MS}^{2}$ architecture and its supporting mechanisms can be found in details in [3]. Figure 1 presents the considered $\mathrm{MS}^{2}$ model topology, which involves three media servers servicing a number of user equipment (UE) via a single access point (AP). The example network includes three uncorrelated paths from the servers to the UE side. A Domain Manager or a Decision Maker (DM) in the $\mathrm{MS}^{2}$ architecture handles the overall service management.

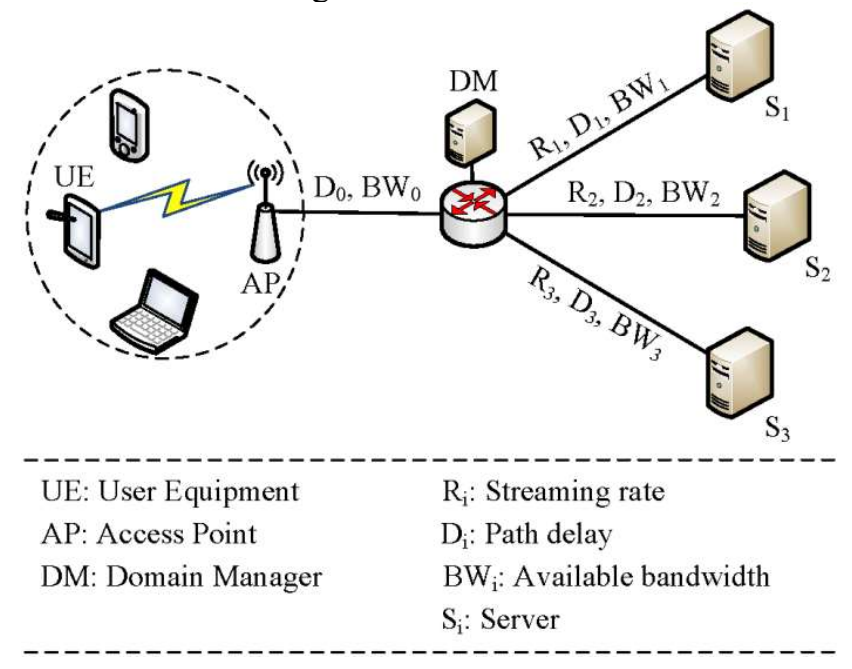

Figure 1. Simplify $\mathrm{MS}^{2}$ model with three servers.

As detailed in [3], $\mathrm{MS}^{2}$ operates following a number of states. In the initial state, the DM sends a request information packet to its management Server [i] with $i=1,2,3$ in this example network; and receives some of reply information packets from the Server[i]. A path delay $\left(D_{i}\right)$ from Server $\left.i\right]$ to the DM is calculated by the DM and then, available bandwidth $\left(B W_{i}\right)$ of the bottleneck link between the DM and the Server $[i]$ is estimated by the information packet size over the path delay. It should be noted that DM usually updates $B W_{i}$ because of fluctuate network traffic. Following the $\mathrm{MS}^{2}$ paper, after having $B W_{i}$ from appropriate servers, the DM continues to calculate: streaming rate $\left(R_{i}\right)$, number of packets $\left(P_{i}^{v}\right)$ to be sent during the monitoring period $(\delta)$, data packet inter-transmission times $\left(\Delta_{i}\right)$, and the time to commence the transmission $\left(\tau_{i}\right)$. Finally, two parameters $R_{i}$ and $\tau_{i}$ are informed to the Server $[i]$, and the Server [i] also continues to calculate $P_{i}^{v}$ and $\Delta_{i}$.

In the working state, the UE sends the IntPk to request content to the DM. The DM bases on the path delay $D_{i}$, make an appropriate delay to forward the IntPk to all servers nearly at the same time. All servers bases on $\tau_{i}, \Delta_{i}$ and $P_{i}^{v}$, make a packet scheduling to send DataPks to the UE. The servers schedule the delivery of DataPks to the UE in a way that the packets of the same content are arriving in order at UE, without high jitter, and most importantly without duplicate packets that could drain up the battery lifetime of the UE [3]. 


\section{MULTI-SOURCE IN THE CONTEXT OF CDN AND CCN}

Content delivery network (CDN) improves the QoS by replicating the most popular content on some specific repositories, as close as possible to users. The content centric networking (CCN) is name-based routing and caching content along the reverse path from server to clients. Both of CDN and CCN are offloading network traffic, reducing end-to-end packet delay. However, not many works address on the comparison between $\mathrm{CDN}$ and $\mathrm{CCN}$.

The general CDN architecture includes four components: origin server, replica server, RequestRouting mechanism $(R e q-R)$ and client. Origin server is a content provider. Replica server maintains a part of catalog contents from origin server. $R e q-R$ re-directs requests from clients to appropriate replica server. If hitting content, contents are replied to clients by replica server. If missing content, requests from UEs are continue to fetch to origin server. Push phase is a process to copy contents from origin server to replica servers. The copied contents are selected as popular contents to enable maximize hitting rate at replica servers. Pull phase is a process that contents are fetched from origin server (or replica servers) to clients. In the context of $\mathrm{MS}^{2}, \operatorname{Req}-R$ is added into domain manager (DM) to enable the delivery of the requests from MSs to replica servers.

\subsection{Non-cooperative Push Phase}

Let $H$ denote hitting rate at replica server in the MS-CDN scheme. We assume that all replica servers have the same capacity of storage including the same popular contents, dubbed "non-cooperative push phase". In this scheme, origin servers have knowledge about content popularity, enabling the most popular contents to be chosen and transferred to all replica servers. We use Pareto distribution as popular contents distribution with a probability distribution function (pdf):

$$
f(x)=\frac{c \cdot a^{c}}{x^{c+1}}
$$

Where $a$ denotes a scale parameter, $c$ denotes a shape parameter, and $x$ denotes content identification with a constraint. Let $N_{f}$ denote a number of the most popular contents can be located in replica servers, and $n_{\text {video }}$ denote the catalog contents (the number of demand videos). Relative cache size is:

$$
R_{\text {cache }}=\frac{N_{f}}{n_{\text {video }}}
$$

Then, hitting rate is calculated as:

$$
H=\sum_{x=a}^{a+N_{f}} \frac{c \cdot a^{c}}{x^{c+1}}=\frac{c}{a} \cdot \sum_{x=a}^{a+N_{f}}\left(\frac{c}{x}\right)^{c+1} \text { with } N_{f} \leq n_{\text {video }}
$$

The number of request during simulation is:

$$
n_{\text {req }}=n_{U E} \cdot \frac{t_{\text {stop }}-t_{\text {start }}}{l_{\text {length }}}
$$

The number of demand video is:

$$
n_{\text {video }}=\max \{x-a+1\} \text {, subject to } n_{\text {req }} \cdot f(x) \geq 1, \forall x \geq a
$$

\subsection{Cooperative Push Phase}

Having described the operation of $\mathrm{MS}^{2}, \mathrm{CDN}$ architectures and principle components. We now present on how the $\mathrm{MS}^{2}$ can be integrated to CCN strategies, dubbed MS-CCN. In MS-CCN scheme, when $\mathrm{CCN}$ nodes are found for the stream of a particular content, they operate as the same fashion of servers in $\mathrm{MS}^{2}$ architecture. As shown in the Fig.2, because of uncorrelated reverse paths from servers to DM, the particular content is fragmented and transmitted as "cooperative push phase" to CCN nodes.

Let $H_{i}$ denote the hitting rate obtained on each path from servers to UE, $R_{\text {Offloading }[i]}$ denotes amount of traffic offloaded on Path $_{[i}$, the network non-congestion condition and bottleneck link utilization on each path will be: 


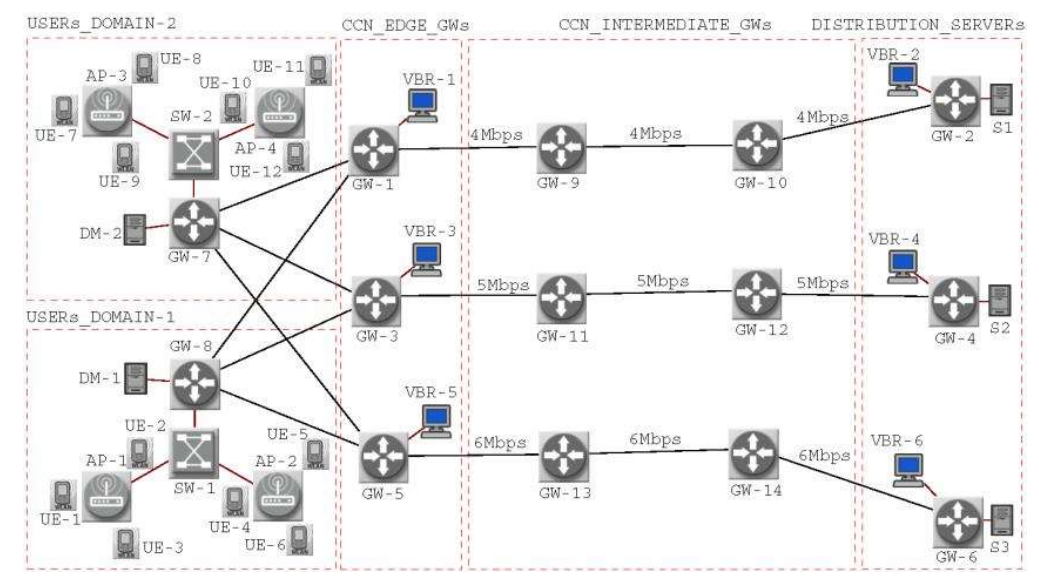

Figure 2. MS-CCN network simulation with three server's distribution.

$$
\begin{aligned}
& R_{\text {Offloading }[i]}=H_{i} \cdot\left(n_{U E} \cdot R_{i}\right), \forall i=1,2, \ldots, n_{S} \\
& B W_{i}>\left(1-H_{i}\right) \cdot\left(n_{U E} \cdot R_{i}\right)+R_{V B R} \\
& \left(1-H_{i}\right) \cdot n_{U E} \cdot\left(\frac{B W_{i}}{\sum_{i=1}^{n_{S}} B W_{i}} \cdot R_{p}\right)+R_{V B R} \\
& U_{\text {Link }[i]}=\frac{\left(W_{i}\right.}{}
\end{aligned}
$$

\subsection{Network architecture}

To evaluate the performance of MS-CDN and MS-CCN, we implemented CDN, CCN, $\mathrm{MS}^{2}$ and conducted simulations using the OPNET Modeler 16.0. In the simulations, CCN is overlayed over the IP layer. Indeed, we integrated the CCN processing modules into all network elements, such as router, UE, server and DM. With every intention to consider a typical Internet network topology, we envision a typical multi-source CCN model with three server's distribution as shown in Fig.2 for the simulation. There are eight UEs requesting different contents and every UE requires seamless video streaming with a constant rate $800 \mathrm{Kbps}$. UEs send randomly the request content around the $50^{\text {th }}$ second after the start of the simulation, e.g. 50+Uniform $(0 ; 10) s$. Videos are streamed from video servers and finally received by CCN processors located inside gateway/router. After executing underlying video caching/replacement policies, CCN processors supply requesting UEs with requested video content when available at CCN nodes. In this paper, we apply FIFO replacement policy to achieve the benefits of $\mathrm{CCN}$.

In order to make different Quality of Experiment (QoE) between the single-source scheme and multi-source scheme, we set up a bottleneck link between server side and client side. Because of the bottleneck link, some of packets may be dropped and cannot reach UE during the network traffic congestion time. UE maintains the time-out to resend the request for the packet loss. Moreover, in order to simulate a realistic background traffic, variable bit rate (VBR) with different shapes of network traffic is also considered between GW-1 and GW-2, generated by VBR-1 and VBR-2. The VBR is combined by two shape of network traffic at the same time, e.g. Uniform $(0.002 ; 0.02)+$ Poisson $(0.02) s$, for intertransmission packets.

The packet size is fixed at $1 K B$. All VBRs are initiated at the $10^{\text {th }}$ second after the start of the simulation and maintained until the end of the simulation. For instance, we consider Pareto with $a=10^{4}$ and $c=10^{2}$ for both math model and simulation model. Hitting rates are compared to check consistency between results. Starting from the simulation, there are 12 UEs sending request content at around 50s. The simulation is terminated at $2000 \mathrm{~s}$. The length of video content is set to $2 \mathrm{~s}$ to speed up simulation time. 


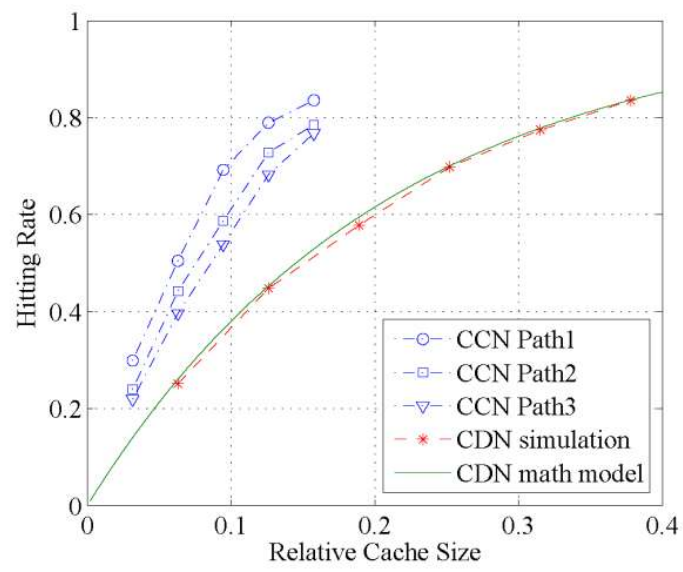

Figure 3. MS-CDN and MS-CCN with three server's distribution.

In the MS-CDN scheme, six scenarios with $N_{f}=30,60,90,120,150$ and 180 , respectively. Under the same network configuration with the MS-CDN scheme, we set up five scenarios with $N_{f}=15,30,45,60$, and 75, respectively for the MS-CCN scheme. From the simulation results in Fig.3, we conclude some important notes:

- Data points of hitting rate in the CDN OPNET simulation match with theoretical curve based on CDN math model, which demonstrates the accuracy of our mathematical model and validating our simulation setup.

- CCN approach always outperforms than CDN approach in the same network simulation. Because of non-cooperative push phase, all of replica servers in CDN have the same contents. In the CCN scheme, the content is distributed over several CCN nodes (located inside edge routers/gateways) which help the $\mathrm{CCN}$ scheme can store more popular contents than CDN scheme.

- However, the replica server of CDN is typical much greater than content store of CCN, then CDN approach may has the same or higher performance than CCN approach. Thus, CDN approach is trade-off cost of the replica server and performance.

\section{MULTIPLE SCENARIOS FOR MS-CCN MODEL EVALUATION}

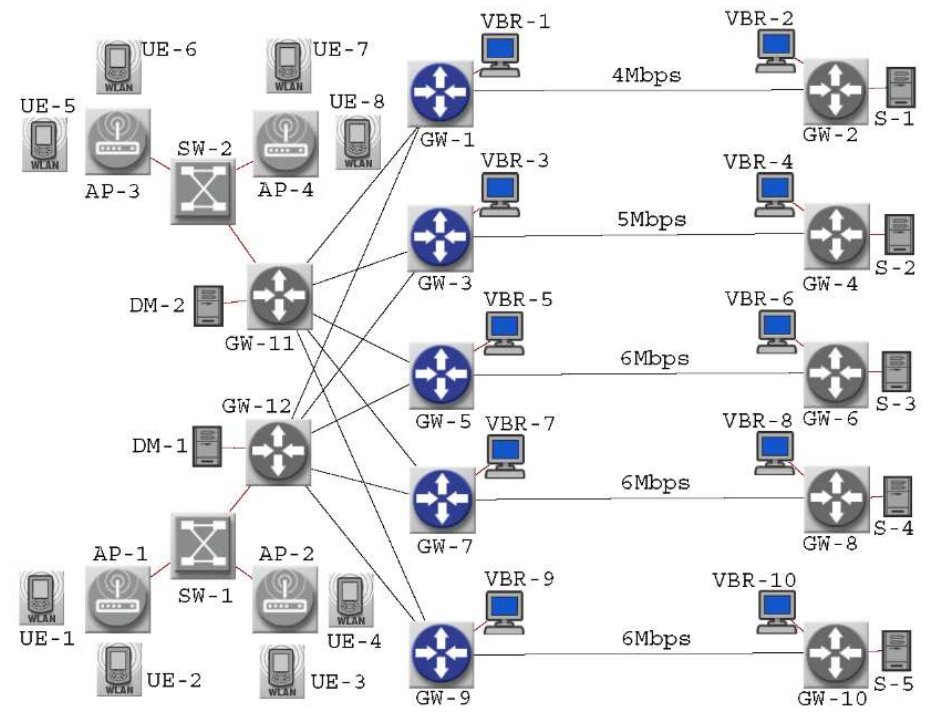

Figure 4. A simplify MS-CCN network simulation with five server's distribution. 
Table 1. Simulation Parameters.

\begin{tabular}{|l|l|l|}
\hline \multicolumn{1}{|c|}{ Name } & \multicolumn{1}{|c|}{ Attribute } \\
\hline \multirow{3}{*}{ Packet types } & Information & $32 \mathrm{~B}$ \\
& Interest (IntPk) & $32 \mathrm{~B}$ \\
& Data (DataPk) & $1 \mathrm{~KB}$ \\
\hline \multirow{5}{*}{ UE } & Buffer size & $100 \mathrm{Pks}$ \\
& Play rate & $100 \mathrm{Pk} / \mathrm{s}$ \\
& Wireless interface & $802.11 \mathrm{~g} @ 54 \mathrm{Mbps}$ \\
& DataPk time-out & $0.6 \mathrm{~s}$ \\
& Start time & $50+\mathrm{Uniform}(0 ; 10) \mathrm{s}$ \\
& Stop time & $2000 \mathrm{~s}$ \\
\hline DM/Server & Monitoring time interval & $0.3 \mathrm{~s}$ \\
\hline \multirow{3}{*}{ Bottleneck links } & Single server & $6 \mathrm{Mbps}$ \\
& Two servers & $5 ; 6 \mathrm{Mbps}$ \\
& Three servers & $4 ; 5 ; 6 \mathrm{Mbps}$ \\
& Four servers & $4 ; 5 ; 6 ; 6 \mathrm{Mbps}$ \\
& Five servers & $4 ; 5 ; 6 ; 6 ; 6 \mathrm{Mbps}$ \\
\hline \multirow{2}{*}{ Other links } & GW-GW & OC-24 \\
& Other links & $1000 \mathrm{Base}-\mathrm{X}$ \\
\hline \multirow{2}{*}{ Contents } & Video size & $200 \mathrm{DataPk} / 2 \mathrm{~s}$ \\
\hline \multirow{2}{*}{ Content Store (CS) } & Popularity characteristic & Pareto $\left(10^{4} ; 10^{2}\right)$ \\
\hline
\end{tabular}

Figure 4 depicts the considered MS-CCN model simulation topology, which includes five servers. As shown in Fig.4, the topology consists of five uncorrelated paths from the servers to the gateway traversing a number of routers/gateways. The simulation network includes two wireless domains. All UEs within the radio range of AP-1 and AP-2 are managed by DM-1. Similarly, UEs, connected to AP-3 and AP-4, are handled by DM-2.

In the envision network in Fig.4, caching contents are deployed at edge gateways for all simulation schemes, e.g. GW-1; GW-3; GW-5; GW-7 and GW-9. Edge caching is of vital importance for the CCN which becomes highly efficient with intelligent caching at edge routers/gateways. This efficiency degrades as caching occurs far from end-users. By caching, some popular video contents are accessed from nearby caches, bypassing bottleneck links. For this reason, the average data packet delays become reduced. To evaluate a spreading of requirement bandwidth through multi-source and disjoint multi-path, some assessment metrics such as: bottleneck link utilization, hitting rate, offloading servers traffic and server responding delay are used in the simulation. Table I lists the rest of the simulation parameters.

Figure 5a compares the bandwidth (BW) utilization on the $6 \mathrm{Mbps}$ bottleneck link under variety multi-source. In the typical single-source $\mathrm{CCN}$ scheme, the single-path link utilization often reaches its maximum capacity, resulting in network congestion. By leverage the concept of $\mathrm{MS}^{2}$, the content delivery is distributed among several servers, e.g. 2, 3, 4, and 5 servers, respectively, with disjoint routing paths to UE. Thus, the minimum available BW requirement on routing paths are reduced quickly, associated with the number of servers. For this reason, the MS-CCN model not only uses less bandwidth from the bottleneck links but also improves the utilization free BW of the entire network.

Figure $5 \mathrm{~b}$ compares among the $\mathrm{CCN}$ performance of different multi-source schemes when the relative cache size is set to 0.1 . In Fig. $5 \mathrm{~b}$, the obtain simulation results show that a better hitting rate can be achieved when increasing the number of server's distribution. When higher number of server's distribution is explored, resulting lower data packets are delivered on each routing path and unfrequently replacements of data packets at caches. Under $\mathrm{MS}^{2}$ streaming flows, CSs from edge CCN nodes located in disjoint multi-path are cooperative in popular content storage. Thus, the hitting rate at CCN node is increased in associate with the number servers 1, 2, 3, 4, and 5, respectively. From the figure, it becomes apparent that this increase in the hitting rate is not linear to increase in the number of servers. 


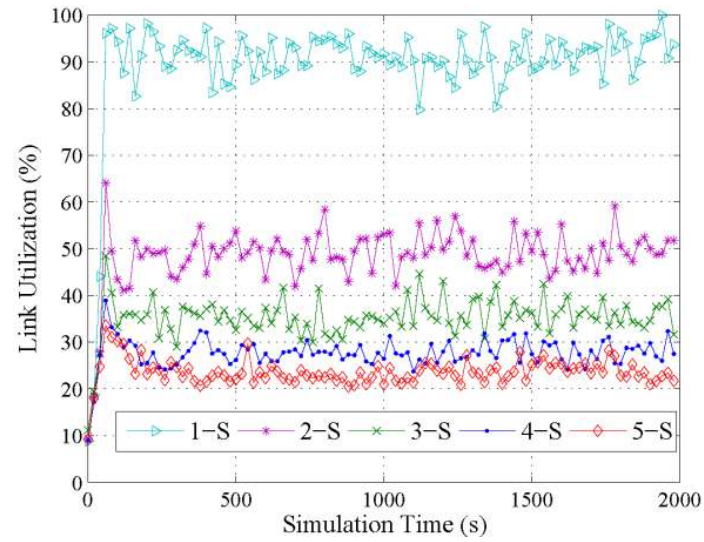

(a) Utilization on the $6 \mathrm{Mbps}$ bottleneck links.

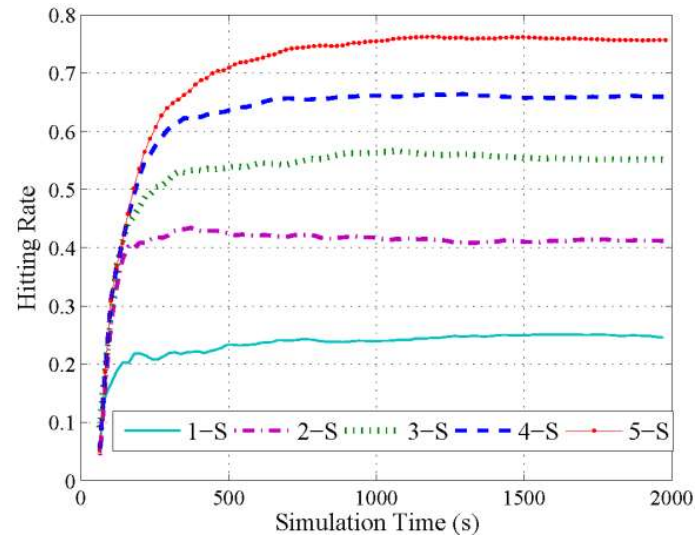

(b) Hitting rate on the $6 \mathrm{Mbps}$ bottleneck links.

Figure 5. Links utilization comparison.

Figure 6a presents the impact of number servers distribution on the total server's load. In CCN strategy, instead of sending all the IntPks to the origin servers, $\mathrm{CCN}$ nodes act as surrogates to original servers and the cached contents are responded to the end users, then offloading the server load. If CCN nodes perform higher hitting rate, lower requested traffic is fetched to servers, then higher percentage of offloading traffic is achieved. Obviously, in the Fig. $6 \mathrm{a}$, the total responding data bit rate sent by servers is reduced in associate with the number of servers 1,2,3,4, and 5, respectively.

Figure $6 \mathrm{~b}$ further compares among the five schemes for different relative cache sizes. From this figure, we realize that higher number of server's distribution exhibits always higher hitting rate in all situations. Furthermore, the figure reveals two important observations. First, when the relative cache size is too small (e.g. 0.025), the increasing number of servers has too few benefit to improve the hitting rate. Second, when the relative cache size is large enough (e.g. 0.2), a high number of server's distribution (e.g. 5 ) is not necessary because the hitting rate is just slightly improvement. There is thus the trade-off between cache volume, number of servers and performance, and there is consequently need to retrieve a suitable value of cache size and servers.

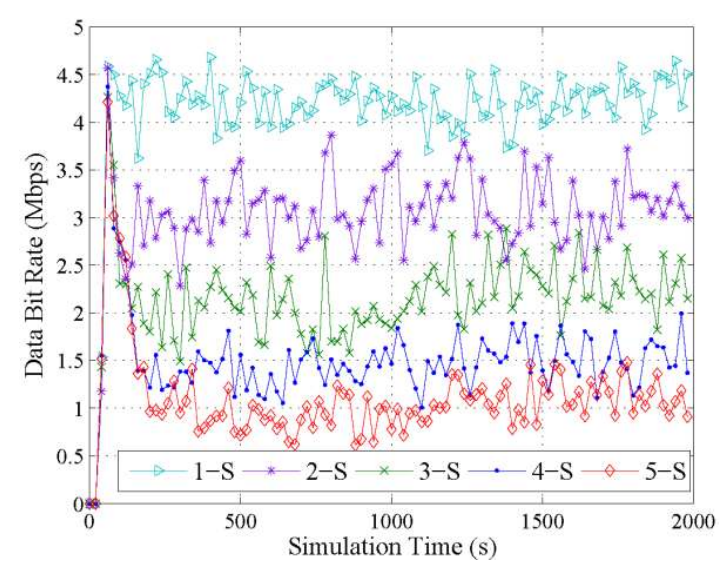

(a) Sum of servers traffic.

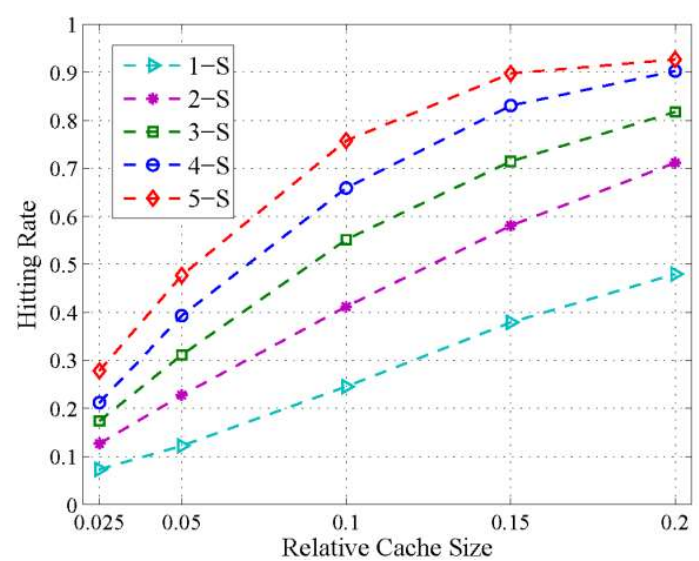

(b) Final state for varying relative cache size.

Figure 6. Content offloading comparison. 


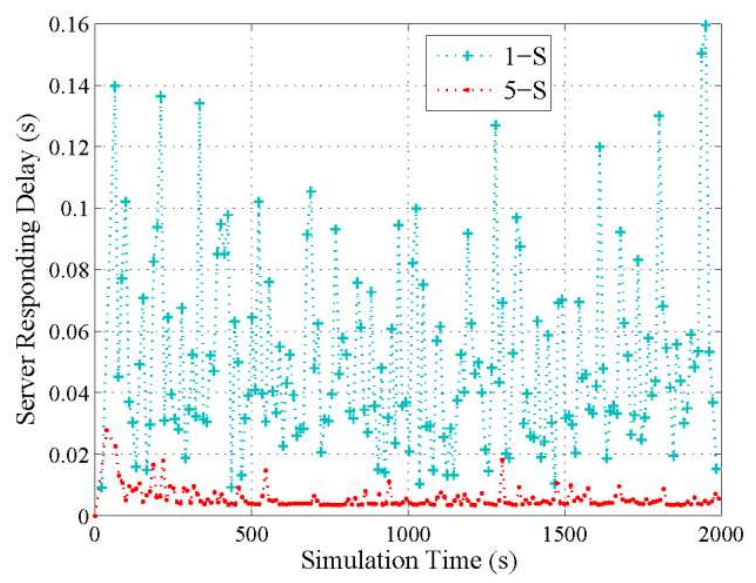

Figure 7. Server responding delay at the UE.

Figure 7 presents the total time elapsed since a UE issues an IntPk requesting a video till it receives the first DataPk of the video. And the relative cache size is set to 0.1 as default in this scheme. As shown in the Fig.7, in the typical single-source CCN scheme, because of low hitting rate on the single CCN node, a lot of DataPks need to be fetched from the single server, passing through bottleneck link. At the congestion time, the delivery delay of DataPks increases, highly dynamic and some packets losses occur. Hence, the responding delay in the single-source $\mathrm{CCN}$ scheme is always higher and varying than the MS$\mathrm{CCN}$ scheme, and that is during entire simulation time. Obviously, in the MS-CCN scheme, when the desired content is cached at several $\mathrm{CCN}$ nodes located on disjoint multi-path to the UE, an entire available cache size increases along with the number of data paths (or the number of server's distribution). For this reason, popular videos can be accessed immediately from nearby nodes with negligible delay. Otherwise, un-popular videos take a delay of transmission through bottleneck links with congestion avoidant.

\section{CONCLUSIONS}

In this paper, we discussed the recently $\mathrm{MS}^{2}$ architecture for mobile multimedia streaming to improve the CCN performance. The resultant architecture, dubbed MS-CCN, efficiently distributes contents available to mobile users and improves the utilization of the overall free bandwidth through disjoin multi-path. This helps in avoiding the access to popular content from far away servers along paths that could otherwise get congested. The performance of MS-CCN was evaluated through computer simulation and compared against the typical single-source CCN. The obtained results demonstrated the effectiveness of MS-CCN with different number of server's distribution schemes. Additional simulation results on video playback quality such as delay and jitter stability also demonstrated that MS-CCN could outperform the typical single-source $\mathrm{CCN}$ in utilizing overall network resources more efficiently. Envisioning such smart caching and predictive available bandwidth form our future goal in this particular research area.

\section{REFERENCES}

[1] Cisco, Cisco Visual Networking Index: Global Mobile Data Traffic Forecast Update, 2014-2019, white paper, Feb. 2015.

[2] P. A. L. Rego, M. S. Bonfim, M. D. Ortiz, J. M. Bezerra, D. R. Campelo and J. N. de Souza, An OpenFlowBased Elastic Solution for Cloud-CDN Video Streaming Service, 2015 IEEE Global Communications Conference (GLOBECOM), San Diego, CA, 2015, pp. 1-7.

[3] T. Taleb and K. Hashimoto, $\mathrm{MS}^{2}$ : A New Real-Time Multi-Source Mobile-Streaming Architecture, IEEE Transaction on Broadcasting, vol. 57, no. 3, pp. 662-673, Sep. 2011. 
[4] C. Li, J. Liu and S. Ouyang, Characterizing and Predicting the Popularity of Online Videos, IEEE Access, vol. 4, pp. 1630-1641, 2016.

[5] V. Jacobson, D. Smetters, J. Thornton, M. Plass, N. Briggs, and R. Braynard, Networking named content, Communication of the ACM, vol. 55, no. 1, pp. 117-124, Jan. 2012.

[6] M. Chen, OPNET Network Simulation, Press of Tsinghua University, ISBN 7-302-08232-4, 2004.

[7] T. Taleb, N. Kato, and Y. Nemoto, Neighbors-Buffering Based Video-on-Demand Architecture, Signal Processing: Image Communication journal, vol. 18, no. 7, pp. 515-526, Aug. 2003.

[8] D. Yun, H. Kim and K. Chung, HTTP adaptive streaming scheme for multi-server environments, 2016 International Conference on Information and Communication Technology Convergence (ICTC), Jeju Island, South Korea, 2016, pp. 717-719.

[9] J. Wu, Z. Zhu, X. Di, Z. Zhang and J. Tian, Multi-path selection and scheduling scheme for multi-description video streaming in wireless multi-hop networks, 2016 International Wireless Communications and Mobile Computing Conference (IWCMC), Paphos, 2016, pp. 970-975.

[10] Y. C. Chen, D. Towsley and R. Khalili, MSPlayer: Multi-Source and Multi-Path Video Streaming, IEEE Journal on Selected Areas in Communications, vol. 34, no. 8, pp. 2198-2206, Aug. 2016.

[11] G. Zhang, H. Li, T. Zhang, D. Li and L. Xu, A multi-path forwarding strategy for content-centric networking, 2015 IEEE/CIC International Conference on Communications in China (ICCC), Shenzhen, 2015, pp. 1-6.

[12] A. Udugama, S. Palipana and C. Goerg, Analytical Characterisation of Multi-path Content Delivery in Content Centric Networks, Conference on Future Internet Communications (CFIC), pp. 1-7, May 2013.

\section{NÂNG CAO HIỆ SUẤT ĐỊNH TUYẾN MẠNG THÔNG QUA ĐỊNH DANH NỘI DUNG DỰA TRÊN PHÂN PHỐI ĐA NGUỒN NỘI DUNG}

Tóm tắt. Ngày nay, định tuyến mạng thông qua định danh nội dung $(\mathrm{CCN})$ trở thành một trong những công nghệ quan trọng trong mạng thế hệ mới. Trong $\mathrm{CCN}$, các nội dung được lưu trữ trên đường định tuyến từ nơi cung cấp về nơi yêu cầu, và có thể được tái sử dụng nhiều lần mà không lấy từ nơi cung cấp ban đầu. Vì vậy, $\mathrm{CCN}$ cải thiện hiệu suất mạng bằng cách giảm thiểu truyền dẫn các nội dung có tính phổ biến. Tuy nhiên, dung lượng lưu trữ tại các phần tử định tuyến bị giới hạn, và nhỏ hơn nhiều so với kích thước của nội dung trên Internet, đặc biệt là khi các ứng dụng đa phương tiện được xem xét. Để giải quyết vấn đề này, bài viết này đề xuất giải pháp dựa trên việc sử dụng phân phối đa nguồn nội dung, ứng dụng vào $\mathrm{CCN}(\mathrm{MS}-\mathrm{CCN})$. Trong mô hình $\mathrm{MS}-\mathrm{CCN}$, bộ nhớ đệm của từng nội dung không giới hạn trong một máy chủ duy nhất mà được phân mảnh từ nhiều máy chủ trên một mạng quy mô lớn. Các mảnh nội dung khác nhau sẽ truyền dẫn trên các đường định tuyến khác nhau và được kết nối lại tại nơi yêu cầu. Các kết quả mô phỏng trong OPNET Modeler cho thấy rằng giải pháp MS-CCN nâng cao hiệu suất sử dụng mạng và chất lượng dịch vụ hơn nhiều lần so với $\mathrm{CCN}$ truyền thống.

Từ khóa. Định tuyến mạng thông qua định danh nội dung, bộ nhớ đệm, đa luồng, giảm tải lưu lượng mạng.

Ngày nhận bài: 27/02/2017

Ngày chấp nhận đăng: 12/06/2017 